
is related to lesser terminal duct lobular unit involution

\title{
of the breast
}

\author{
Clara Bodelon (iD ${ }^{1 凶}$, Hannah Oh $^{1,2}$, Andriy Derkach ${ }^{1}$, Joshua N. Sampson ${ }^{1}$, Brian L. Sprague ${ }^{3}$, Pamela Vacek $^{3}$, Donald L. Weaver $^{3}$, \\ Shaoqi Fan ${ }^{1}$, Maya Palakal ${ }^{1}$, Daphne Papathomas ${ }^{1}$, Jackie Xiang ${ }^{1}$, Deesha A. Patel ${ }^{1}$, Laura Linville ${ }^{1}$, Susan E. Clare ${ }^{4}$, \\ Daniel W. Visscher ${ }^{5}$, Carolyn Mies ${ }^{6}$, Stephen M. Hewitt ${ }^{7}$, Louise A. Brinton ${ }^{1}$, Anna Maria V. Storniolo ${ }^{8}, \mathrm{Chunyan}^{10} \mathrm{BD}^{9,10}$, \\ Stephen J. Chanock (D) ${ }^{1}$, Montserrat Garcia-Closas (D) ${ }^{1}$, Gretchen L. Gierach (D) ${ }^{1,12}$ and Jonine D. Figueroa (iD)
}

Terminal duct lobular units (TDLUs) are the predominant anatomical structures where breast cancers originate. Having lesser degrees of age-related TDLU involution, measured as higher TDLUs counts or more epithelial TDLU substructures (acini), is related to increased breast cancer risk among women with benign breast disease (BBD). We evaluated whether a recently developed polygenic risk score (PRS) based on 313-common variants for breast cancer prediction is related to TDLU involution in the background, normal breast tissue, as this could provide mechanistic clues on the genetic predisposition to breast cancer. Among 1398 women without breast cancer, higher values of the PRS were significantly associated with higher TDLU counts $(P=0.004)$, but not with acini counts $(P=0.808)$, in histologically normal tissue samples from donors and diagnostic BBD biopsies. Mediation analysis indicated that TDLU counts may explain a modest proportion ( $\leq 10 \%)$ of the association of the 313-variant PRS with breast cancer risk. These findings suggest that TDLU involution might be an intermediate step in the association between common genetic variation and breast cancer risk.

npj Breast Cancer (2020)6:41 ; https://doi.org/10.1038/s41523-020-00184-7

\section{INTRODUCTION}

Terminal duct lobular units (TDLUs) are the milk-producing structures of the breast and the predominant anatomical structures from which breast cancers originate ${ }^{1}$. Higher number of TDLUs and higher number of acini (TDLU epithelial substructures) indicate lesser TDLU involution. Among benign breast disease (BBD) patients, having reduced levels of TDLU involution is associated with increased risk of subsequent breast cancer ${ }^{2,3}$. Measures of TDLU involution are thought to reflect a global process occurring throughout the breast ${ }^{2,4,5}$. Two studies from European and Asian populations have also found greater TDLU involution surrounding ER-positive compared with triple negative breast cancers, primarily for acini measures ${ }^{6,7}$. While several hormonal and lifestyle breast cancer risk factors are known to affect TDLU involution ${ }^{8}$, how genetic risk factors relate to TDLU involution is not well understood.

We previously examined whether 62 established breast cancer susceptibility loci were associated with TDLU involution and found limited evidence for an association ${ }^{9}$. Recently, a polygenic risk score (PRS) for the prediction of breast cancer has been derived using 313 common variants from the largest available international genome-wide association dataset ${ }^{10}$. Any observed relationships between the 313-variant PRS and TDLU involution may provide mechanistic clues on the genetic predisposition to breast cancer. ER-specific PRS have also been recently developed ${ }^{10}$.
Investigating whether ER-specific PRS are related to measures of TDLU involution could inform whether lobular involution shows differential relationships for genetic susceptibility predisposition to breast cancer subtypes.

Here, we assessed the relation between the 313-variant breast cancer PRS and TDLU involution in 1,398 women with histologically normal donated tissues in the Susan G. Komen Tissue Bank (KTB) at the Indiana University Simon Cancer Center $(n=1,089)^{11}$ and in the background, normal tissue of biopsies among participants diagnosed with $\mathrm{BBD}$ in the $\mathrm{NCl}$ Breast Radiology Evaluation and Study of Tissues (BREAST) Stamp Project $(n=309)^{12}$.

\section{RESULTS}

Patients characteristics

Characteristics of the participants are shown in Table 1. The majority of the women were premenopausal (76\%), parous (58\%), and $22 \%$ had a family history of breast cancer in a first degree relative. Approximately $68 \%$ of the women had TDLUs observed, with a median of $8.5 \mathrm{TDLUs} / 100 \mathrm{~mm}^{2}$ and 12 acini/TDLU. Agerelated TDLU involution metrics were similar in the KTB and the BREAST Stamp studies.

\footnotetext{
${ }^{1}$ Division of Cancer Epidemiology and Genetics, National Cancer Institute, Bethesda, MD, USA. ${ }^{2}$ Division of Health Policy and Management, College of Health Sciences, Korea University, Seoul, Korea. ${ }^{3}$ University of Vermont College of Medicine and Vermont Cancer Center, Burlington, VT, USA. ${ }^{4}$ Department of Surgery, Feinberg School of Medicine, Northwestern University, Chicago, IL, USA. ${ }^{5}$ Department of Laboratory Medicine and Pathology, Mayo Clinic, Rochester, MN, USA. ${ }^{6}$ Department of Pathology and Laboratory Medicine, University of Pennsylvania, Philadelphia, PA, USA. ' Laboratory of Pathology, Center for Cancer Research, National Cancer Institute, Bethesda, MD, USA. ${ }^{8}$ Susan G. Komen Tissue Bank at the Indiana University Simon Cancer Center, Indianapolis, IN, USA. 'Department Internal Medicine, Division of Medical Oncology, College of Medicine, University of Kentucky, Lexington, KY, USA. ${ }^{10}$ Markey Cancer Center, University of Kentucky, Lexington, KY, USA. ${ }^{11}$ Usher Institute of Population Health Sciences and Informatics and Cancer

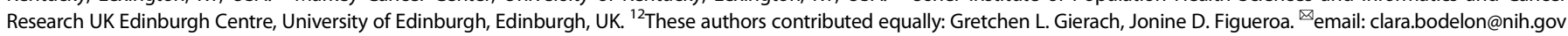


Table 1. Descriptive statistics of TDLU involution measures.

\begin{tabular}{|c|c|c|c|}
\hline Characteristic & $\begin{array}{l}\text { Overall } \\
(N=1398)\end{array}$ & $\begin{array}{l}\text { Komen } \\
\text { Tissue Bank } \\
(N=1089)\end{array}$ & $\begin{array}{l}\text { BREAST Stamp } \\
\text { Project } \\
(N=309)\end{array}$ \\
\hline \multicolumn{4}{|l|}{ Age (years), $n$ (\%) } \\
\hline$<30$ & $318(22.7)$ & $318(29.2)$ & 0 \\
\hline $30-39$ & $234(16.7)$ & $234(21.5)$ & 0 \\
\hline $40-49$ & $431(30.8)$ & $274(25.2)$ & $157(50.8)$ \\
\hline $50-59$ & $301(21.5)$ & $186(17.1)$ & $115(37.2)$ \\
\hline$\geq 60$ & $114(8.2)$ & $77(7.1)$ & $37(12.0)$ \\
\hline \multicolumn{4}{|l|}{ Menopausal status, $n$ (\%) } \\
\hline Premenopausal & $1,053(76.4)$ & $847(79.2)$ & $206(66.7)$ \\
\hline Postmenopausal & $325(23.6)$ & $222(20.8)$ & $103(33.3)$ \\
\hline \multicolumn{4}{|l|}{ Parity, $n$ (\%) } \\
\hline Nulliparous & $581(41.6)$ & 507 (46.6) & $74(23.9)$ \\
\hline Parous & $817(58.4)$ & $582(53.4)$ & $235(76.1)$ \\
\hline \multicolumn{4}{|l|}{ Family history, n (\%) } \\
\hline No & $1,081(77.6)$ & $853(78.3)$ & $228(75.0)$ \\
\hline Yes & $312(22.4)$ & $236(21.7)$ & $76(25.0)$ \\
\hline \multicolumn{4}{|l|}{ Observed TDLUs, $n(\%)$} \\
\hline No & $455(32.5)$ & 369 (33.9) & $86(27.8)$ \\
\hline Yes & $943(67.5)$ & $720(66.1)$ & $223(72.2)$ \\
\hline TDLU count, median (IQR) & $3(0-11)$ & $3(0-11)$ & $4(0-14)$ \\
\hline $\begin{array}{l}\mathrm{TDLU} /\left(100 \mathrm{~mm}^{2}\right) \\
\text { median (IQR) }\end{array}$ & $8.5(0.0-31.0)$ & $8.4(0.0-31.8)$ & $9.7(0.0-26.7)$ \\
\hline $\begin{array}{l}\text { Median acini count per TDLU, } \\
\text { median (IQR) }\end{array}$ & $12(7-18.5)$ & $12(7-19)$ & $11(7.1-16.5)$ \\
\hline $\begin{array}{l}\text { Median acini count per TDLU } \\
\text { (including women with } 0 \\
\text { TDLUs), median (IQR) }\end{array}$ & $7(0-14.5)$ & $7(0-15)$ & $8(0-13.5)$ \\
\hline $\begin{array}{l}\text { (TDLU counts per unit area) * } \\
\text { (acini count/TDLU), median } \\
(\text { IQR) }\end{array}$ & $2.4(0.8-6.4)$ & $2.5(0.9-7.3)$ & $2.1(0.6-4.7)$ \\
\hline $\begin{array}{l}\text { (TDLU count per unit area)* } \\
\text { (acini count/TDLU) (including } \\
\text { women with } 0 \text { TDLUs), } \\
\text { median (IQR) }\end{array}$ & $0.8(0-4.2)$ & $0.8(0-4.2)$ & $0.9(0-3.2)$ \\
\hline
\end{tabular}

Association between the polygenic risk score for the prediction of breast cancer and TDLU involution

Greater values of the PRS were statistically significantly associated with higher TDLU counts ( $P=0.004$; Table 2$)$. Specifically, women in the top 25th percentile of polygenic risk had 33\% increased odds (95\% Cl: 1.06-1.65) of having greater TDLU counts compared with women in the bottom 25th percentile. Results were similar, although attenuated, after adjusting for the visually assessed proportion of fibroglandular (nonfatty) tissue. The PRS was not associated with acini counts/TDLU $(P=0.808)$. With respect to the 313 loci that formed the PRS, we found limited evidence for their individual associations with TDLU involution measures (Supplemental Data 1). Analyses stratified by study population suggested a stronger association in the tissue donors (KTB) compared to those diagnosed with BBD (BREAST Stamp) (data not shown). ERpositive and ER-negative PRS were both positively associated with TDLU count ( $P=0.005$ for ER-positive and negative) but not with acini count/TDLU ( $P=0.861$ and $P=0.715$, respectively).

\section{Mediation analysis}

Mediation analysis was used to estimate the percent of the association between the PRS and breast cancer risk that might be explained by TDLU count (Fig. 1, Table 3). Ideally such an analysis would be conducted in a study where the PRS, TDLU measures, and breast cancer risk are all available. However, no study, to our knowledge, has all three measures. Therefore, we developed a novel approach where we used information from different sources

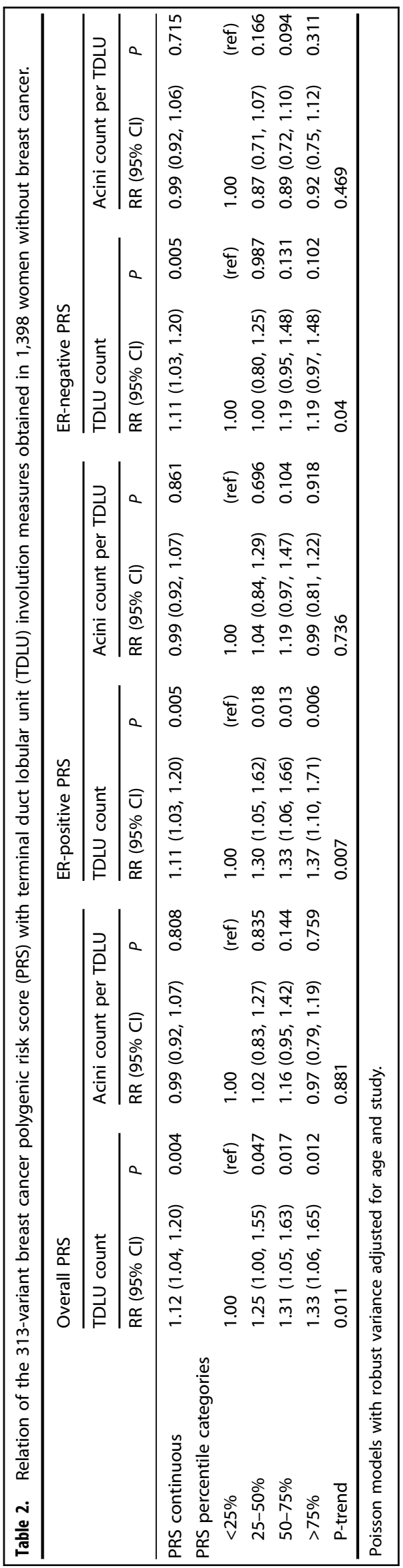

Published in partnership with the Breast Cancer Research Foundation 


\section{Overall PRS-Breast cancer association}
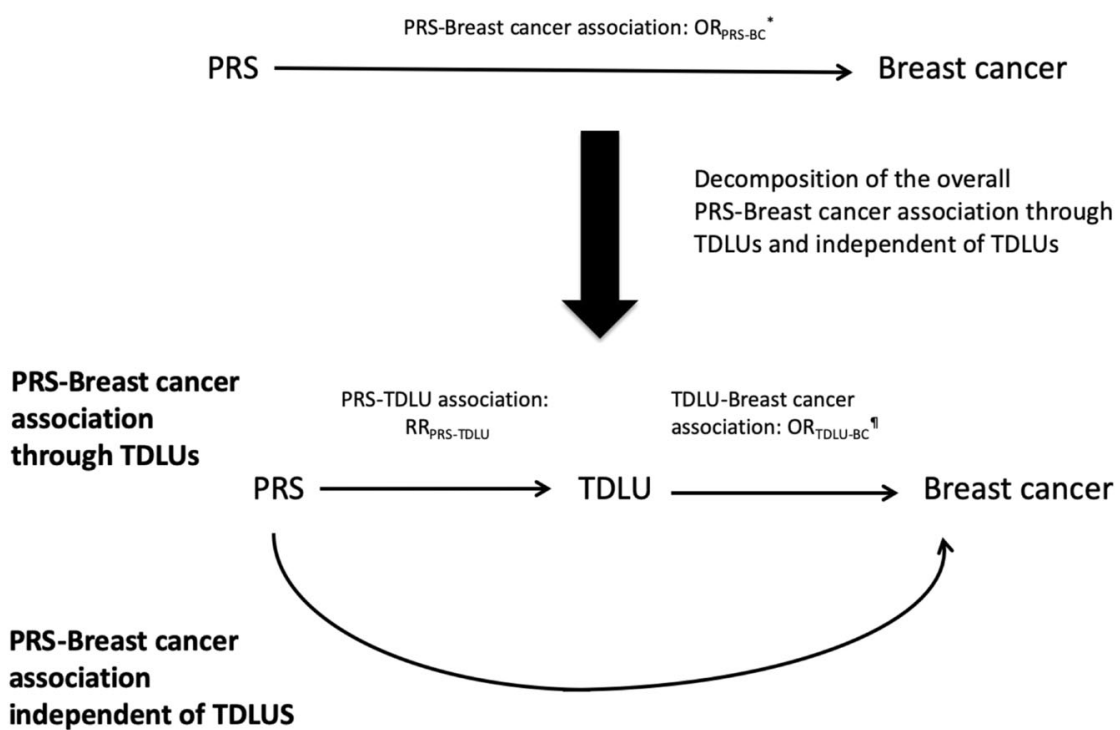

"Previously reported using data from the Breast Cancer Association Consortium; Mavaddat et al. ${ }^{8}$

"TDLU-breast cancer association based on data presented in Figueroa et al. ${ }^{11}$

Fig. 1 Relationship between the 313-varieant breast cancer polygenic risk score (PRS), terminal duct lobular unit (TDLU) measures and risk of breast cancer. Estimated percent of the association between the 313-variant breast cancer polygenic risk score (PRS) and breast cancer (BC) risk explained by terminal duct lobular unit (TDLU) counts.

Table 3. Percent of the association between the 313-variant breast cancer polygenic risk score (PRS) and breast cancer risk explained by TDLU counts.

\begin{tabular}{|c|c|c|c|c|c|c|c|}
\hline PRS & $\begin{array}{l}\text { Breast } \\
\text { cancer } \mathrm{OR}^{\mathrm{a}, \mathrm{b}}\end{array}$ & $\mathrm{RR}^{\mathrm{a}}$ & $(95 \% \mathrm{Cl})$ & $P$ value & $\begin{array}{l}\% \text { explained by TDLU } \\
\text { counts and } 95 \% \mathrm{Cl} \text { if } \\
\mathrm{OR}_{\mathrm{TDLU}-\mathrm{BC}}=1.25^{\mathrm{C}}\end{array}$ & $\begin{array}{l}\% \text { explained by TDLU } \\
\text { counts and } 95 \% \mathrm{Cl} \text { if } \\
\mathrm{OR}_{\mathrm{TDLU}-\mathrm{BC}}=1.35^{\mathrm{C}}\end{array}$ & $\begin{array}{l}\% \text { explained by TDLU } \\
\text { counts and } 95 \% \mathrm{Cl} \text { if } \\
\mathrm{OR}_{\text {TDLU-BC }}=1.45^{\mathrm{C}}\end{array}$ \\
\hline ER+ & 1.68 & 1.11 & $(1.03,1.20)$ & 0.005 & $3.47(1.63,4.54)$ & $5.05(2.36,7.09)$ & $6.52(2.92,9.46)$ \\
\hline ER- & 1.45 & 1.11 & $(1.03,1.20)$ & 0.005 & $5.28(2.40,7.48)$ & $7.49(3.41,11.04)$ & $9.54(4.10,14.40)$ \\
\hline
\end{tabular}

to estimate the percent explained. The relationship between the PRS and breast cancer risk was obtained from the Breast Cancer Association Consortium ${ }^{10}$; the estimates of the association between TDLU counts (continuous) and breast cancer risk were based on previous results from the Mayo Benign Breast Disease Cohort $^{13}$; and the present analysis provided the relationship between the PRS and TDLU counts. Using this approach, we estimated that $\sim 4$ to $7.4 \%$ of the relationship between the PRS and breast cancer risk would be explained by TDLU counts, if the odds ratio for the association between TDLU counts and breast cancer risk was between 1.25 and 1.45 based on previous results from the Mayo Benign Breast Disease Cohort.

\section{DISCUSSION}

In this study, we assessed the relation between the 313-variant breast cancer PRS and measures of TDLU involution and found that higher values of the PRS were significantly associated with higher TDLU counts in histologically normal/benign breast biopsies. Although individual single nucleotide polymorphisms
(SNPs) in the PRS have been associated with some breast cancer risk factors (e.g., mammographic breast density ${ }^{14}$ ), TDLU involution is the first breast cancer risk factor found to be significantly associated with the 313-variant PRS. Lack of TDLU involution and elevated mammographic breast density are positively correlated histologic and radiologic measures, respectively, of breast tissue composition that are thought to provide independent information about breast cancer risk in women with $\mathrm{BBD}^{15}$. Mammographic density was not readily available in the KTB participants, and therefore, we did not have enough statistical power to evaluate the relationship between the 313-PRS and mammographic density in our study. If future studies identify a relation of the 313-PRS with mammographic density, then one could investigate interrelationships between the PRS, TDLU involution and mammographic density in relationship to breast cancer risk.

Our result relating the 313-PRS with TDLU counts corroborates previous findings showing that family history of breast cancer is associated with increased TDLU counts ${ }^{8}$. Similarly, the lack of observed association between the PRS and acini counts/TDLU is consistent with studies that did not find associations with family 
history $^{8}$, suggesting that there is not a strong heritable component for the acinar TDLU substructures/size of TDLUs, which may instead be influenced by hormonal/environmental factors (e.g. menopausal hormone use).

Strengths of this study include the well-characterized epidemiologic and genetic data for study participants with standardized, reproducible measures of lobular involution in nonmalignant breast tissues. We also applied an innovative analytical approach to estimate the percent of the association between PRS and breast cancer risk potentially explained by TDLU counts. A limitation of the current study is the lack of replication of our findings. Our study is based on observational assessment of TDLU involution. Although we have demonstrated strong within- and between-rater reliability for these TDLU metrics ${ }^{5,8}$, computational pathology approaches may offer future opportunities to advance etiologic studies examining complex relationships between genetic susceptibility, breast tissue composition, and breast cancer risk. In addition, we focused our assessment of involution in the background normal tissue, excluding any observed BBD lobules. Even so, prior work suggests that TDLU involution in histologically normal breast tissue may reflect a global process occurring throughout the breast ${ }^{2,4,5}$. Finally, despite doubling the sample size from our previous analysis ${ }^{9}$, additional samples may be required to detect associations between individual genetic variants and TDLU metrics.

In conclusion, we observed a statistically significant association between a recently developed 313-variant PRS for the prediction of breast cancer and TDLU counts, suggesting that TDLU involution may mediate relationships underlying common genetic susceptibility and breast cancer risk. Future studies may further investigate the use of the PRS for the prediction of TDLU involution, with potential applications in studies that lack measures of lobular involution.

\section{METHODS}

\section{Study population and samples}

The study population has been previously described ${ }^{9}$. Briefly, the KTB is an annotated biobank, which has recruited healthy women who provided demographic, lifestyle, and cancer-related information via selfadministered questionnaire, blood samples and normal breast tissues. Up to four breast tissue cores were obtained from the upper outer quadrant of the breast using a standardized technique with a 10-gauge vacuum-assisted biopsy. One sample was formalin-fixed and paraffinembedded and stained with hematoxylin and eosin (H\&E). Whole blood samples were collected using Vacuette ${ }^{\circledR}$ EDTA tubes. For this study, a $50 \mu \mathrm{l}$ aliquot of samples were reconstituted at the Cancer Genomics Research laboratory (Leidos Biomedical Research, Inc., Frederick, MD) for genotyping. Details of the KTB have been described elsewhere (http:// komentissuebank.iu.edu/) ${ }^{11}$. The current analysis was restricted to 1507 participants recruited from January 10, 2009 through September 14, 2012, aged 18-91 years, that were eligible for genotyping. Informed consent was obtained from all participants to use of their specimens and questionnaire data for research. Approval for this study was obtained from the Indiana University Institutional Review Board (IRB) and the National Institutes of Health Office of Human Subjects Research (NIH OHSR \#4508).

The NCI BREAST Stamp Project is a cross-sectional study of mammographic density conducted among women, aged $40-65$ years, who were referred for diagnostic image-guided breast biopsy from 2007 through 2010 at the University of Vermont College of Medicine and University of Vermont Medical Center. Demographic, lifestyle, and breast cancer risk factor information were collected via a self-administered questionnaire and a supplementary telephone interview. Participants underwent clinicallyindicated breast biopsies, which were formalin-fixed paraffin-embedded blocks and H\&E stained. Whole blood samples and mouthwash samples were collected as previously described ${ }^{16}$. Blood and mouthwash samples were processed at the University of Vermont General Clinical Research Center. Leukocyte DNA was extracted from blood clots using phenol chloroform, and DNA was isolated from buccal cells using Gentra Puregene Buccal Cell Kits (Qiagen). The current analysis was restricted to 450 women who had eligible samples for genotyping. All women provided written informed consent and the study was approved by the IRBs at the University of Vermont and the $\mathrm{NCl}$.

\section{TDLU involution assessment}

H\&E slides were scanned for image analysis on Digital Image Hub software (SlidePath/Leica, Dublin, Ireland). The total tissue area $\left(\mathrm{mm}^{2}\right)$ on the slides was outlined using the lasso tool in Digital Image Hub and measured. The study pathologist measured the number of TDLUs ("TDLU count"). Acini count per TDLU was quantified using the TDLU analyzer software ${ }^{17,18}$ as previously described ${ }^{9}$. A high intra-observer agreement (Spearman's $r>$ 0.90 ) for the TDLU measures was previously reported ${ }^{5,8}$.

\section{OncoArray genotyping}

DNA samples from 1957 women in the two studies (1507 from the KTB and 450 BREAST Stamp project) and 23 quality control samples were genotyped on the Illumina OncoArray chip $^{19}$ at the Cancer Genomics Research laboratory (Frederick, MD, USA). Genotypes were generated using a cluster file provided by the Genetic Associations and Mechanisms in Oncology Consortium. Details of the OncoArray SNP selection and genotyping calling have been described elsewhere 19,20. Briefly, 50\% of the SNPs for the OncoArray were selected to provide high coverage for imputation of common variants. The other $50 \%$ of SNPs were selected from lists supplied by investigators based on consortia decisions for different diseases. Of the 568,712 variants selected for genotyping on the OncoArray, 533,631 were successfully manufactured on the array (including 778 duplicate probes). Of these 533,631 variants, 38,868 were defined as problematic SNPs by the Consortium QC guideline, thus were excluded from the current analyses.

We further excluded samples and SNPs based on the criteria defined by the consortium QC guideline. Through a stepwise filtration, we excluded SNPs and samples with a call rate $<95 \%$ and samples with extreme heterozygosity $(>0.4$ or $<0.05)$ within each study. We also excluded 9 unexpected duplicates with genotype concordance rate $>99 \%$, excluded one nonfemale subject with $\mathrm{X}$ chromosome heterozygosity close to zero and 66 first-degree relatives based on identity by descent analysis. Using a set of population informative SNPs ${ }^{20}$ and the 1000 Genome phase 3 data, we identified 98 subjects of non-European descent whom we excluded from the analyses.

After genotyping, subjects were further excluded if they had a personal history of in situ or invasive breast cancer, has missing tissue area, pregnant at the time of blood draw, were previous donors (in the KTB), and/or currently taking hormone therapy, leaving an analytic population of 1398 women.

\section{Imputation}

All samples were imputed using the October 2014 (version 3) release of the 1000 Genomes Project dataset as the reference panel and using a twostage imputation approach using SHAPEIT $2^{21}$ and IMPUTE ${ }^{22}$ version 2 , as in Michailidou et al. ${ }^{23}$, run on the NIH High-Performance Computing Biowulf cluster computing (http://hpc.nih.gov). The imputation was done in 5-MB around intervals around each of the 313 loci in the PRS ${ }^{10}$. Imputation quality as assessed by IMPUTE version 2 quality score was $>0.80$ and $r^{2}>$ 0.3 for all loci.

\section{Statistical analysis}

Analyses were conducted combining data from the KTB study and BREAST Stamp Project. The PRS for overall and ER-specific disease was computed using the 313 loci as described in Mavaddat et al..$^{10}$ and the log of effects sizes as weights. We standardized the PRS to have units of standard deviation (SD). With respect to the subtype-specific PRS, we used the weights from the hybrid method in Mavaddat et al., as this method was found to have the best performance.

Similar to our previous analyses ${ }^{9}$, we used Poisson regression models with robust variance to compute per-SD relative risks and $95 \%$ confidence intervals for the association of TDLU measures (i.e., TDLU count and acini count per TDLU) with the PRS. The PRS was modeled as a continuous variable and as a categorical variable based on quartiles computed in the entire study population. An offset variable was included in the model to account for the tissue area on the slide. We used the Wald test to assess whether a linear trend between the PRS and morphometric TDLU measures was present. Similar analyses were used to compute per-allele RR (Supplementary Tables 1-2). We also fitted linear 
regression models to confirm that the associations were not driven by the Poisson model assumptions, similar to our previous analysis ${ }^{9}$, and results were similar. All multivariable models were adjusted for study population (KTB or BREAST Stamp Project) and age. Sensitivity analysis were also carried out by adjusting for family history and visually assessed proportion of fibroglandular tissue. No significant differences were observed. We also conducted stratified analyses by study and by family history (data not shown).

The estimation of the mediating effect was done using the procedure described by Imai et al. ${ }^{24}$ and the $95 \% \mathrm{Cl}$ were obtained by the bootstrap procedure with 10,000 resamples. For this procedure, we assumed that the relationship between the PRS and TDLU could be described by Poisson regression (see above) and that the parameters could be estimated using data from this study. Furthermore, we assumed that the joint effect between PRS and TDLU count on breast cancer could be described by logistic regression Eq. (1):

$$
\begin{aligned}
P(\text { Breast cancer }= & 1 \mid \text { TDLU count, PRS })=\exp \left(\beta_{0}+\beta \times\right. \text { TDLU count } \\
& +\gamma \times P R S) /\left(1+\exp \left(\beta_{0}+\beta \times \text { TDLU count }+\gamma \times P R S\right)\right),
\end{aligned}
$$

with parameters estimated so that the three marginal relationships were appropriately constrained (i.e. $\mathrm{P}$ (Breast cancer $=1 \mid \mathrm{PRS}$ ) agreed with Mavaddat et al. ${ }^{10}, \mathrm{P}($ Brest cancer $=1 \mid$ TDLU count) agreed with the assumed value from Figueroa et al. ${ }^{13}$, and f(TDLU |PRS) agreed with results from our study). The constrained model was fit using approaches recently developed by Chatterjee et al. ${ }^{25}$.

All statistical tests were two-sided and $P$ values $<0.05$ were considered statistically significant. Analyses were conducted using R 3.5.2 on a Mac platform.

\section{Reporting summary}

Further information on research design is available in the Nature Research Reporting Summary linked to this article.

\section{DATA AVAILABILITY}

The data generated and analyzed using the KTB specimens will be returned to the KTB and it will available through their virtual repository (https://virtualtissuebank.iu. edu/). To protect patient privacy of the data generated in the STAMP project, deidentified data generated and analyzed will be available on request from Dr. Gretchen L. Gierach (gierachg@mail.nih.gov). The data generated and analysed during this study are described in the following data record: https://doi.org/10.6084/ m9.figshare. $12293108^{26}$. The PRS data are in the R file "BC.impute.313.Rdata". The BREAST Stamp Project data are not publicly available because the informed consent signed by the patients did not include public data sharing. To request these data, contact Dr. Gretchen Gierach (gierachg@mail.nih.gov). However, the Susan G. Komen Tissue Bank (KTB) data are available through the Susan G. Komen Tissue Bank (KTB)'s virtual repository (http://virtualtissuebank.iu.edu) and the phenotype and polygenic risk scores are available from the dbGaP repository under the following accession ID: https://identifiers.org/dbgap:phs002062.v1.p1 ${ }^{27}$.

\section{CODE AVAILABILITY}

The code developed during the current study are available upon reasonable request.

Received: 10 December 2019; Accepted: 6 August 2020;

Published online: 07 September 2020

\section{REFERENCES}

1. Russo, J., Hu, Y. F., Yang, X. \& Russo, I. H. Chapter 1: Developmental, cellular, and molecular basis of human breast cancer. JNCI Monographs 2000, 17-37 (2000).

2. Milanese, T. R. et al. Age-related lobular involution and risk of breast cancer. J. Natl. Cancer Inst. 98, 1600-1607 (2006).

3. Baer, H. J. et al. Lobule type and subsequent breast cancer risk: results from the Nurses' Health Studies. Cancer 115, 1404-1411 (2009).

4. Vierkant, R. A. et al. Lobular involution: localized phenomenon or field effect? Breast Cancer Res. Treat. 117, 193-196 (2009).

5. Gierach, G. L. et al. Relationship of terminal duct lobular unit involution of the breast with area and volume mammographic densities. Cancer Prev. Res. (Philos.) 9, 149-158 (2016).
6. Yang, X. R. et al. Analysis of terminal duct lobular unit involution in luminal A and basal breast cancers. Breast Cancer Res. 14, R64 (2012).

7. Guo, C. et al. Age-related terminal duct lobular unit involution in benign tissues from Chinese breast cancer patients with luminal and triple-negative tumors. Breast Cancer Res. 19, 61 (2017).

8. Figueroa, J. D. et al. Terminal duct lobular unit involution of the normal breast: implications for breast cancer etiology. J. Natl. Cancer Inst. 106, dju286, https://doi.org/10.1093/jnci/dju286 (2014).

9. Bodelon, C. et al. Association between breast cancer genetic susceptibility variants and terminal duct lobular unit involution of the breast. Int J. Cancer 140, 825-832 (2017).

10. Mavaddat, N. et al. Polygenic risk scores for prediction of breast cancer and breast cancer subtypes. Am. J. Hum. Genet 104, 21-34 (2019).

11. Sherman, M. E. et al. The Susan G. Komen for the cure tissue bank at the IU Simon Cancer Center: a unique resource for defining the "Molecular Histology" of the breast. Cancer Prev. Res. 5, 528-535 (2012).

12. Gierach, G. L. et al. Comparison of mammographic density assessed as volumes and areas among women undergoing diagnostic image-guided breast biopsy. Cancer Epidemiol. Biomark. Prev. 23, 2338-2348 (2014).

13. Figueroa, J. D. et al. Standardized measures of lobular involution and subsequent breast cancer risk among women with benign breast disease: a nested case-control study. Breast Cancer Res. Treat. 159, 163-172 (2016).

14. Stone, J. et al. Novel associations between common breast cancer susceptibility variants and risk-predicting mammographic density measures. Cancer Res. 75, 2457-2467 (2015).

15. Ghosh, K. et al. Independent association of lobular involution and mammographic breast density with breast cancer risk. J. Natl. Cancer Inst. 102, 1716-1723 (2010).

16. Garcia-Closas, M. et al. Collection of genomic DNA from adults in epidemiological studies by buccal cytobrush and mouthwash. Cancer Epidemiol. Biomark. Prev. 10, 687-696 (2001).

17. Rosebrock, A. et al. Quantitative analysis of TDLUs using adaptive morphological shape techniques. Proc. SPIE 8676, 86760N (2013).

18. Khodr, Z. G. et al. Circulating sex hormones and terminal duct lobular unit involution of the normal breast. Cancer Epidemiol. Biomark. Prev. 23, 2765-2773 (2014).

19. Amos, C. I. et al. The OncoArray Consortium: a network for understanding the genetic architecture of common cancers. Cancer Epidemiol. Biomark. Prev. 26, 126-135 (2017).

20. $\mathrm{Yu}, \mathrm{K}$. et al. Population substructure and control selection in genome-wide association studies. PLOS ONE 3, e2551 (2008).

21. O'Connell, J. et al. A general approach for haplotype phasing across the full spectrum of relatedness. PLoS Genet 10, e1004234 (2014).

22. Howie, B. N., Donnelly, P. \& Marchini, J. A flexible and accurate genotype imputation method for the next generation of genome-wide association studies. PLoS Genet 5, e1000529 (2009).

23. Michailidou, K. et al. Association analysis identifies 65 new breast cancer risk loci. Nature 551, 92-94 (2017).

24. Imai, K., Keele, L. \& Tingley, D. A general approach to causal mediation analysis. Psychol. Methods 15, 309-334 (2010).

25. Chatterjee, N., Chen, Y.-H., Maas, P. \& Carroll, R. J. Constrained maximum likelihood estimation for model calibration using summary-level information from external big data sources. J. Am. Stat. Assoc. 111, 107-117 (2016).

26. Bodelon, $C$. et al. Metadata record for the manuscript: Polygenic risk score for the prediction of breast cancer is related to lesser terminal duct lobular unit involution of the breast. figshare. https://doi.org/10.6084/m9.figshare.12293108 (2020).

27. dbGaP. https://identifiers.org/dbgap:phs002062.v1.p1 (2020).

\section{ACKNOWLEDGEMENTS}

This study was supported by the Intramural Research Program of the Division of Cancer Epidemiology and Genetics of the US National Cancer Institute. Breast Cancer Research Stamp Funds (awarded to M.E. Sherman and L.A. Brinton) and cooperative agreement U01CA70013 (P.M. Vacek, D.L. Weaver) from the National Cancer Institute funded some of the data collection for this study. The efforts of Drs. Sprague, Vacek, and Weaver were supported in part by cooperative agreement U01 CA196383 from the National Cancer Institute. The content of this publication does not necessarily reflect the views or policies of the Department of Health and Human Services, nor does mention of trade names, commercial products, or organizations imply endorsement by the U.S. Government. Data from the Susan G. Komen Tissue Bank at the IU Simon Cancer Center were used in this study. We thank contributors, including Indiana University who collected data used in this study, as well as donors and their families, whose help and participation made this work possible. 


\section{AUTHOR CONTRIBUTIONS}

C.B., H.O., G.L.G., J.D.F. designed the study. C.B., H.O., A.D., J.N.S., M.G.C., G.L.G., J.D.F. lead the analysis and interpretation of the data. G.L.G., B.L.S., P.V., D.L.W, S.F, M.P., D. P., J., X., D.A.P., L.L., S.E.C, D.W.V., C.M., S.M.H., L.A.B., A.M.V.S., C.H., S.J.C. provided histological and genetic data acquisition. All authors read and approved the final draft of the manuscript. All authors are accountable for all aspects of the work. G.L.G. and J.D.F. contributed equally to this work as senior authors.

\section{COMPETING INTERESTS}

The authors declare no competing interests.

\section{ADDITIONAL INFORMATION}

Supplementary information is available for this paper at https://doi.org/10.1038/ s41523-020-00184-7.

Correspondence and requests for materials should be addressed to C.B.

Reprints and permission information is available at http://www.nature.com/ reprints
Publisher's note Springer Nature remains neutral with regard to jurisdictional claims in published maps and institutional affiliations.

cc (i) Open Access This article is licensed under a Creative Commons Attribution 4.0 International License, which permits use, sharing, adaptation, distribution and reproduction in any medium or format, as long as you give appropriate credit to the original author(s) and the source, provide a link to the Creative Commons license, and indicate if changes were made. The images or other third party material in this article are included in the article's Creative Commons license, unless indicated otherwise in a credit line to the material. If material is not included in the article's Creative Commons license and your intended use is not permitted by statutory regulation or exceeds the permitted use, you will need to obtain permission directly from the copyright holder. To view a copy of this license, visit http://creativecommons. org/licenses/by/4.0/.

This is a U.S. government work and not under copyright protection in the U.S.; foreign copyright protection may apply 2020 